\title{
Broadband light output enhancement for scintillator using whispering-gallery modes in nanospheres
}

\author{
Zhichao Zhu', Bo Liu', , Chuanwei Cheng ${ }^{1}$, Hong Chen', Mu Gu', Yasha Yi, ${ }^{2,3}$, and Rihua Mao ${ }^{4}$ \\ ${ }^{1}$ Shanghai Key Laboratory of Special Artificial Microstructure Materials and Technology, School of Physical Science and Engineering, \\ Tongji University, Shanghai 200092, P.R. China \\ ${ }^{2}$ Integrated Nano Optoelectronics Laboratory, Department of Electrical and Computer Engineering, University of Michigan, Dearborn, \\ MI 48128, USA \\ ${ }^{3}$ Materials Processing Center, Massachusetts Institute of Technology, Cambridge, MA 02139, USA \\ ${ }^{4}$ Laboratory for Advanced Scintillation Materials and Performance, Shanghai Institute of Ceramics, Chinese Academy of Sciences, \\ Shanghai 201800, P.R. China
}

Received 15 January 2014, revised 10 March 2014, accepted 17 March 2014

Published online 17 April 2014

Keywords light extraction, photonic structures, scintillators, whispering-gallery modes

*Corresponding author: e-mail lbo@tongji.edu.cn, Phone: +86 21 65989875, Fax: +86 2165980219

An array of monolayer of periodic nanospheres is used to efficiently extract the light from a scintillator of high refractive index with broadband capability. Compared with the plain sample without nanospheres, the enhancement of wavelength- and angleintegrated luminescence by 72 and $68 \%$ can be achieved under the excitation of ultraviolet and $\gamma$-ray radiation, respectively. The whispering-gallery modes for the individual sphere and the periodic arrangement are responsible for the enhancement of light extraction. Such a photonic structure is advantageous over the traditional two-dimensional photonic crystal.
1 Introduction Scintillators can be defined as materials that absorb incident particles (such as electrons, protons, neutrons) or high-energy photons (such as $\mathrm{X}$-ray, $\gamma$-ray) and convert the deposited energy efficiently into a number of photons in the visible or near-visible range, which can be detected by photomultipliers or photodiodes. By this method, the detection of particles or high-energy photons can be achieved. Therefore, scintillators play an important role in radiation detection systems with applications in highenergy physics experiments and nuclear medical imaging areas. A high light output is vital to improve the sensitivity, spatial resolution and energy resolution in detection systems [1]. For instance, with the improved sensitivity, we can apply a lower radiation dose during the medical imaging procedure (such as X-ray CT). As a result, the cancer risks due to the radiation exposure for patients would be correspondingly decreased [2]. In spite of the high internal quantum efficiency for most practical scintillators, the light-extraction efficiency is rather low due to the total internal reflection. For example, the extraction efficiency of $\mathrm{Bi}_{3} \mathrm{Ge}_{4} \mathrm{O}_{12}$ (BGO) scintillator (refractive index $n=2.15$ ) from one side of the crystal/air interface is as low as 5.4\%, according to an approximate formula $\left(1 / 4 n^{2}\right)$.

In order to improve the extraction efficiency, several nanostructured scintillators have recently been proposed. Phase-separated scintillators with light-guiding properties have been proposed as promising pixilated scintillators in digital X-ray imaging systems [3, 4]. Two-dimensional photonic crystal slabs prepared by electron beam lithography have been used to improve the light extraction for heavy inorganic scintillators [1,5]. Biologically inspired moth-eyelike nanophotonic structures have been utilized to enhance the light extraction for $\mathrm{Lu}_{2} \mathrm{SiO}_{5}$ : $\mathrm{Ce}$ (LSO) scintillator [6]. These demonstrations of light extraction enhancement of scintillators are stimulating interest in the development of nanostructured scintillator. Similarly, in the field of lightemitting diodes (LEDs), many efforts have also been devoted to improve the light extraction by using nanostructures such as photonic crystals [7-10], graded-refractive-index antireflection coatings [11], nanoparticle stacks [12], and cone-shaped nanostructures [13]. When we design a nanostructured scintillator for a practical application, 
several issues have to be taken into account. First, the bandwidth of the emission spectra for scintillators is generally rather broad. For example, the full widths of half-maximum (FWHMs) of emission spectra of LSO and BGO are 80 and $150 \mathrm{~nm}$, respectively. A large area of several square centimeters is often required in a practical detection system. In order to efficiently absorb the high-energy photons or particles, sufficient thickness ranging from millimeters to centimeters is usually necessary.

An array of periodic nanospheres exhibits whisperinggallery modes (WGMs) due to Mie resonance of individual spheres and Bragg diffraction arising from the periodic arrangement [14]. A wavelength-scale dielectric sphere with low-order resonances shows broadband characteristics for optical response [15], which is advantageous to the coupling with broadband emission of scintillators [16, 17]. An array of periodic nanospheres with WGMs has also shown an outstanding ability to enhance the absorption in a broad spectrum for solar cells [18-20]. Additionally, an array of nanospheres with a large area can be easily prepared by selfassembled methods [21]. In this paper, we demonstrate the significant enhancement of light extraction for BGO scintillator in a broad emission spectrum using a monolayer of a periodic array of polystyrene (PS) nanospheres. The improved scintillation performance is presented and the physical mechanism for light extraction is discussed in detail.

2 Experimental method and simulation A commercially available BGO single-crystal scintillator was cut and polished into $3 \mathrm{~mm} \times 10 \mathrm{~mm} \times 20 \mathrm{~mm}$ pieces. The monolayers of 500-nm diameter PS nanospheres with a refraction index of 1.59 (Duck Scientific Corporation) were coated onto the BGO single crystal surface using a modified self-assembly method [22]. The surface topography was measured with a Hitachi S-4800 field-emission scanning electron microscope. The emission spectra were recorded by a fiber spectrometer with a FFT-CCD (PG2000-Pro-Ex, Ideaoptics Co.) under the excitation wavelength of $266 \mathrm{~nm}$ from an UV laser (MPL-F-266, CNI Co.). The samples were fixed on a bracket that can rotate and display the scale of the emerging angle. The excitation light was illuminated on the back surface without structure and the emitted light was detected from the surface covered with PS nanospheres. Light output measurements were performed by using a HAMAMATSU R2059 PMT with a bialkali cathode. The samples were close to the detection window of PMT without using wrapping and coupling of optical glue. A collimated ${ }^{137} \mathrm{Cs} \gamma$-ray source with $662 \mathrm{keV}$ was applied to excite the samples.

The numerical simulations for the transmission spectra and the spatial distributions of electric-field intensity were performed based on a rigorous coupled wave analysis (RCWA) method and a three-dimensional finite-difference time-domain (FDTD) method. The periodic boundary conditions were used. In the simulations for the enhancement ratios of light extraction in Fig. 5, the multireflection by the lower interface between scintillator and air and the multi- diffraction by the monolayer of PS nanospheres were also taken into account.

3 Results and discussion The scheme of light extraction by a monolayer of PS nanospheres is illustrated in Fig. 1a. The self-assembled layer on the BGO scintillator surface forms a hexagonal-close-packed (HCP) structure with two directions of $\Gamma-\mathrm{M}$ and $\Gamma-\mathrm{K}$ indicated in Fig. 1b. The top-view SEM images in Fig. 1c prove the prepared sample as a HCP structure with the diameter of $500 \mathrm{~nm}$ for individual nanospheres. In order to analyze the effect of the monolayer of nanospheres on light extraction, the numerical simulations for transmission at the maximum emission wavelength of $510 \mathrm{~nm}$ are shown in Fig. $2 \mathrm{a}$ and b for s- and p-polarization, respectively. For the BGO with plain surface (reference sample), the transmission drops to zero beyond the critical angle of $27.7^{\circ}$. After being covered with the monolayer of nanospheres, it is fortunate to find that two extra transmission peaks appear at about $30^{\circ}$ and $40^{\circ}$ for both polarizations. This implies that the incident light beyond the critical angle can be partly extracted, leading to an enhanced extraction efficiency.

In order to distinguish the different mechanisms for the two peaks, we also present the calculated transmission of BGO covered by an array of square pillars with the same lattice structure and lattice constant, which is a kind of common two-dimensional photonic crystal slab. The individual square pillar with a dimension of $250 \times 250 \times 500 \mathrm{~nm}^{3}$ is assumed to be absent of WGMs. It is found that beyond the critical angle the array of square pillars also gives rise to the peak at about $30^{\circ}$ but does not produce the peak at about
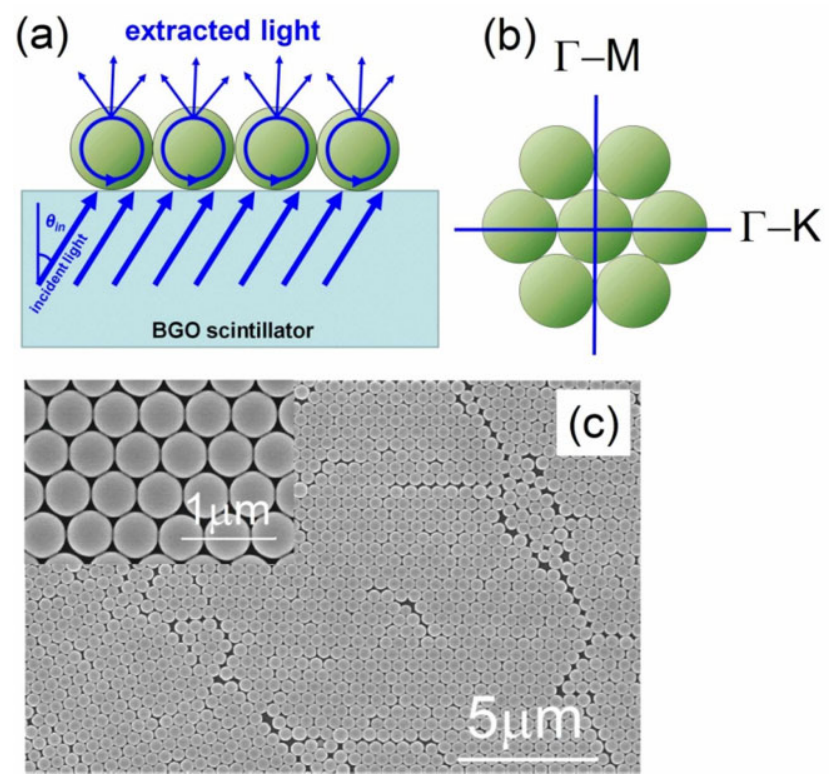

Figure 1 (a) Scheme of the light extraction for a BGO scintillator by a monolayer of PS nanospheres. (b) Sketch of the two different directions of the hexagonal-close-packed structure. (c) Top-view SEM images of the surface of BGO covered with PS nanospheres $(d=500 \mathrm{~nm})$ with two different resolutions. 

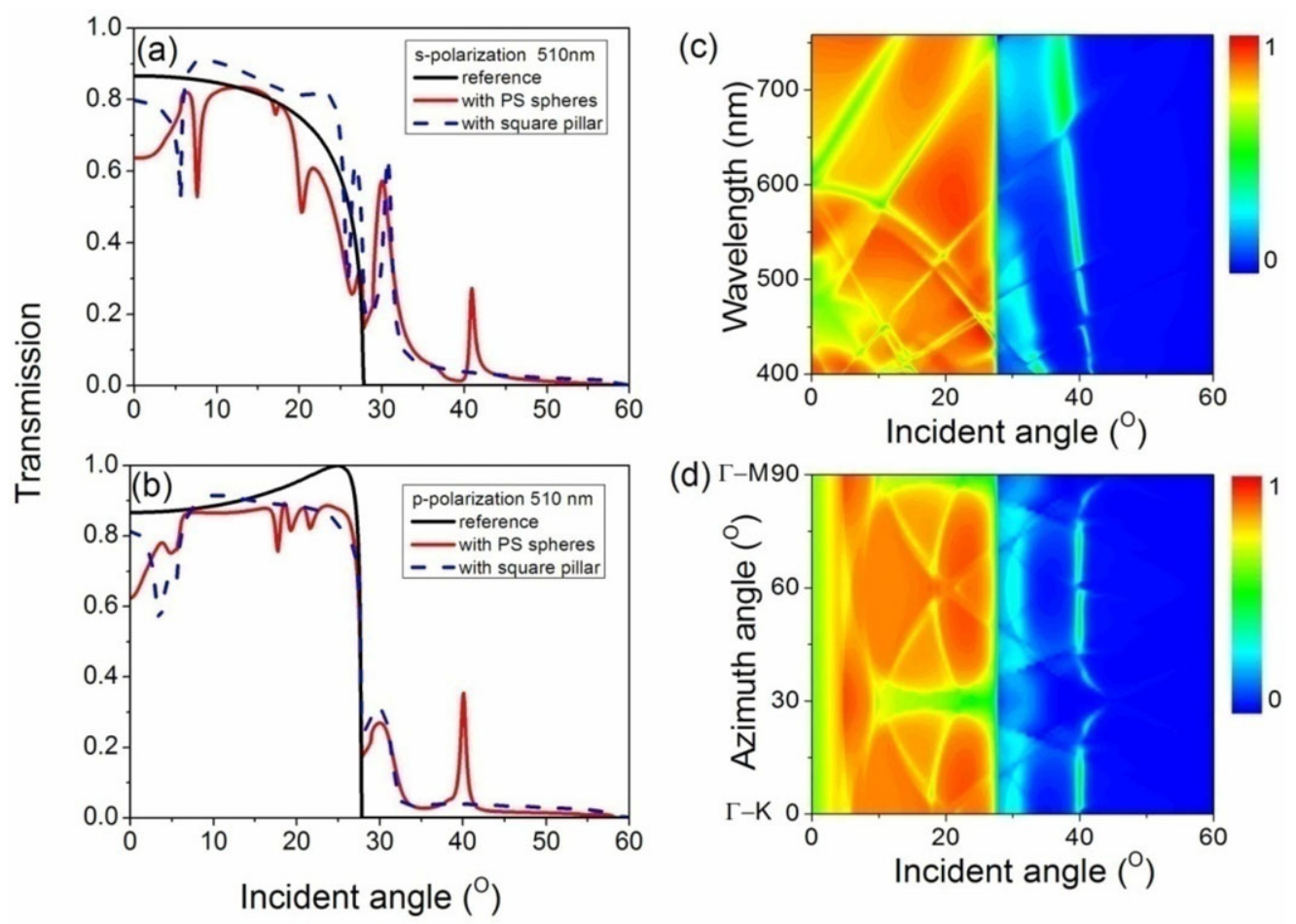

Figure 2 Numerical simulations of transmission spectra with the wavelength of $510 \mathrm{~nm}$ for plain BGO (black solid lines), BGO covered with a monolayer of PS nanospheres with the diameter of $500 \mathrm{~nm}$ (red solid lines), and BGO covered with an array of square pillars with the same structures as the monolayer of PS spheres (blue dashed lines). The simulations with s- and p-polarizations are shown in (a) and (b), respectively. (c) Transmission spectra as a function of wavelength and incident angle for p-polarization. (d) Transmission spectra with the wavelength of $510 \mathrm{~nm}$ as a function of azimuth angle and incident angle.

$40^{\circ}$ for both polarizations. Therefore, it is concluded that the peak at about $30^{\circ}$ could originate from the diffraction of the evanescent field near the interface due to the total internal reflection, which behaves as the light extraction by a common two-dimensional photonic crystal slab [5]. However, the peak at about $40^{\circ}$ is highly correlated with the WGMs that belong to the natural characteristic of nanospheres. The WGMs formed by the coupling of the spheres with the evanescent field near the interface can be further diffracted into far field by the periodic structure, thus giving rise to an additional light extraction. In order to observe the bandwidth for the peaks at about $30^{\circ}$ and $40^{\circ}$, the wavelength-dependent transmission spectra for p-polarization are shown in Fig. 2c. It is found that the considerable broad bandwidth of 400 to $700 \mathrm{~nm}$ and a relatively narrow band of 400 to $500 \mathrm{~nm}$ correspond to the peaks at about $30^{\circ}$ and $40^{\circ}$, respectively. The dispersion relation of decreased peak angle with increasing wavelength is also observed. The azimuthal angle dependence of transmission spectra from the direction of $\Gamma-\mathrm{K}$ to $\Gamma-\mathrm{M}$, shown in Fig. $2 \mathrm{~d}$, indicates that the two peaks at $30^{\circ}$ and $40^{\circ}$ appear for the most of azimuth angles. The enhanced extraction by WGMs exhibits broadband and omnidirection characteristics. Therefore, there is evidence to indicate that the array of monolayer nanospheres with WGMs is advantageous over a common two-dimensional photonic crystal slab for the light extraction.
To further interpret the nature of the modes associated with the peaks in Fig. 2, the simulated spatial distributions of electric-field intensity for a cross section in the middle of a sphere in $\Gamma-\mathrm{K}$ direction are shown in Fig. 3a-d. The distributions of electric-field intensity with wavelengths and incident angles of $694 \mathrm{~nm}, 37.4^{\circ}, 558 \mathrm{~nm}, 39.2^{\circ}$ and $500 \mathrm{~nm}, 40^{\circ}$, shown in Fig. 3a-c, exhibit strong confinement of electric-field intensity inside a sphere, suggesting evident characteristics of WGMs. In contrast, as shown in Fig. 3d, the distribution of electric-field intensity with wavelength and incident angle of $510 \mathrm{~nm}, 30^{\circ}$ does not show any confinement inside a sphere. As a consequence, it is further proved that the transmission peak at about $40^{\circ}$ is associated with the WGMs [23].

The extraction process involving the WGMs can be understood as follows. When the incident light reaches the interface at a certain angle beyond the critical angle, the evanescent field near the interface can be coupled into the array of nanospheres, forming the WGMs that can propagate in the plane of the layer of spheres as a dielectric waveguide due to the coupling among these adjacent spheres $[24,25]$. The light in guided modes would be subsequently diffracted into the far field by the periodic structure as leaky modes. As a result, the incident light beyond the critical angle can be finally out-coupled as farfield emission. Therefore, it is clearly indicated that both the WGMs and the periodic structures play critical roles in light 

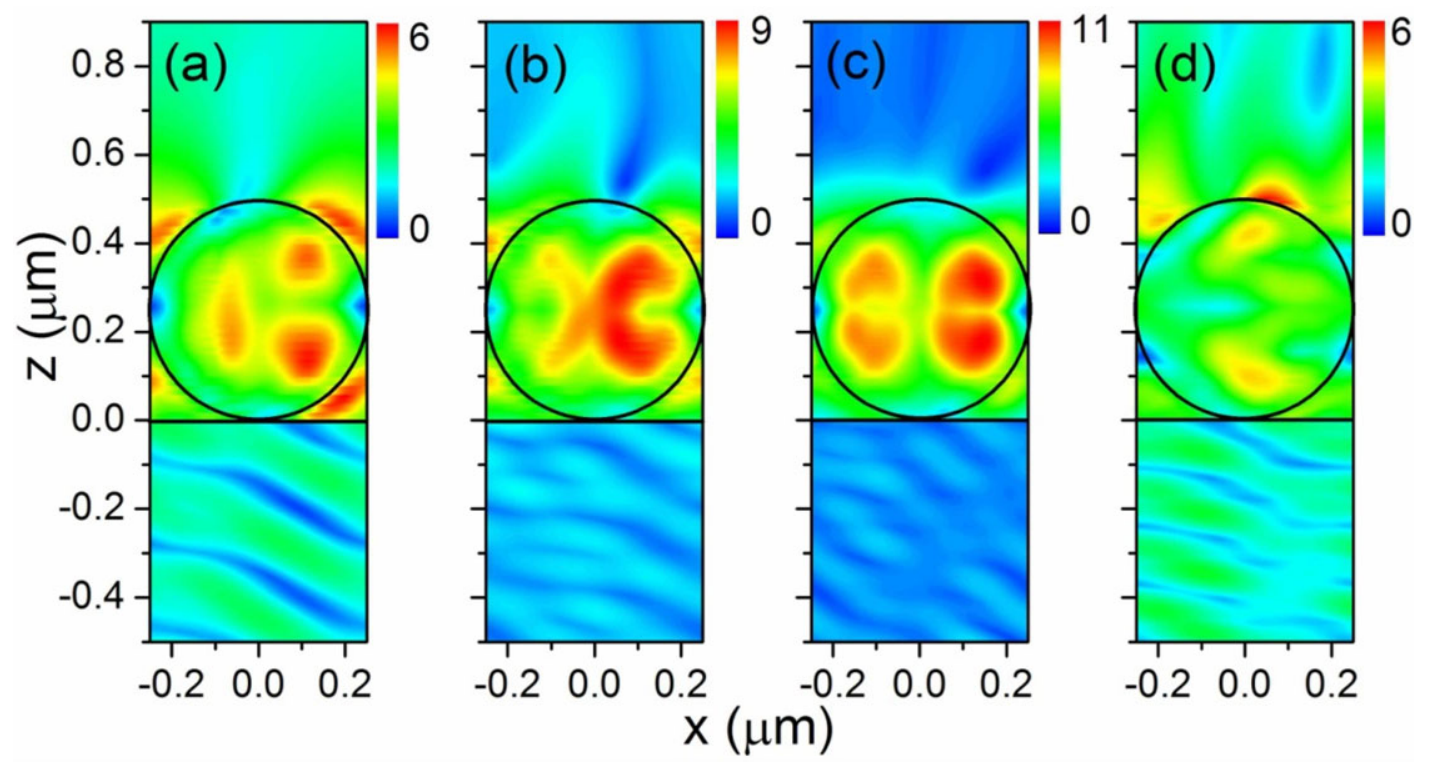

Figure 3 Simulations of spatial distributions of electric-field intensity for BGO covered with a monolayer of PS nanospheres for p-polarization. The wavelengths and incident angles are (a) $694 \mathrm{~nm}, 37.4^{\circ}$, (b) $558 \mathrm{~nm}, 39.2^{\circ}$, (c) $500 \mathrm{~nm}, 40^{\circ}$, and (d) $510 \mathrm{~nm}, 30^{\circ}$. The black circle represents the site of a nanosphere and the negative value in $z$ coordination represents the scintillator.

extraction. Additionally, it is worthwhile to note that below the critical angle several dips appear in the transmission spectra shown in Fig. 2. These dips result in some extra diffracted light back into the crystal. At first glance, the backdiffracted light below the critical angle reduces the light extraction. However, the situation is not severe because the back-diffracted light can be reflected by the back side of the crystal and then be re-extracted by the structures, which could strengthen the ultimate extraction efficiency.

Figure $4 \mathrm{a}$ and $\mathrm{b}$ shows the photoluminescence (PL) spectra of BGO with a monolayer of PS nanospheres $(d=500 \mathrm{~nm})$ and reference sample, respectively. The emission spectra of the reference sample show a very wide band peaked at $510 \mathrm{~nm}$, ranging from 400 to $700 \mathrm{~nm}$. After covering with the monolayer of PS spheres, evident enhancements are observed. Furthermore, the enhancements are strongly dependent on the wavelength and emergence angle. The enhancement ranges from 1.5 to 2.1 depending on the wavelength and the emergence angle. The wavelengthand angle-integrated enhancement reaches $72 \%$. In order to obtain an optimal extraction efficiency, it is required that the diameter should be small enough to ensure that only low order WG modes can be excited, and at the same time it should be large enough to ensure that the diffraction can take place effectively. As a result, experimental and simulated results suggest that the optimal diameter is close to the wavelength involved [17].

The angular profile of emission at 510 and $620 \mathrm{~nm}$ shown in Fig. 4c and d exhibits an approximate Lambertian pattern for the reference sample [26]. While, the emission intensities at 510 and $620 \mathrm{~nm}$ for BGO with nanospheres are significantly increased in the whole emergence angle with the strongest enhancement in the normal direction, making the angular profile deviate from the Lambertian pattern. The directivity of emission is dependent on the diffraction of the structure of the periodic array. This angle-dependent enhancement would be advantageous to the practical applications because only those photons emitted along with the direction of the window of a detector can contribute to the detection. Therefore, from the viewpoint of application, it is necessary to carefully design the structure for scintillators according to the layout of a system for a certain purpose of radiation detection.

In order to characterize the scintillation performance, the light output excited by $662-\mathrm{keV} \gamma$-rays from a ${ }^{137} \mathrm{Cs}$ source is shown in Fig. 4e. The measurements were carried out without using optical grease or wrapping. The energy spectrum reflects the wavelength and angle integration of scintillation photons. For the photopeak, the channel number (the light output) of the structured BGO is larger by $34 \%$ than that of the reference sample. It should be noted that the structured BGO is covered with the PS nanospheres over half the area. Therefore, a corrected enhancement of light output is expected to be about $68 \%$. As for the measurement of PL spectra described above, a mask was used to ensure that the surface covered by PS nanospheres with the same area was exposed. Therefore, the enhancement in PL spectra is in good agreement with that of gamma-energy spectra, suggesting that the enhancement results from the improved light extraction that is independent on the excitation types. It is interesting to observe that the energy resolution for the photopeak is improved from $33.6 \%$ (reference sample) to $30.3 \%$ (sample with PS nanospheres) deduced from the experiment data using Gaussian fitting. The improved energy resolution resulting from the enhancement of light extraction would increase the number of photons towards the photodetector and thus improve the contribution of detector 


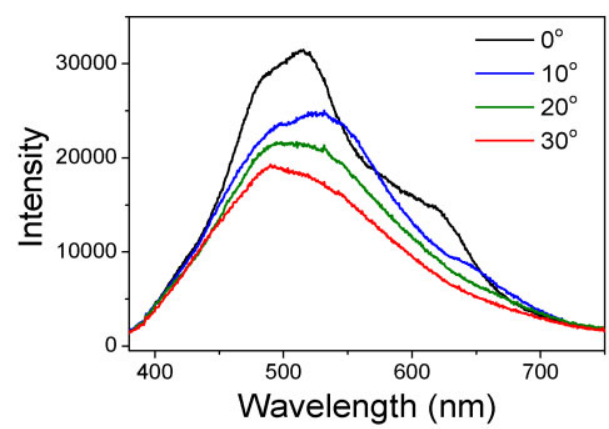

(a)

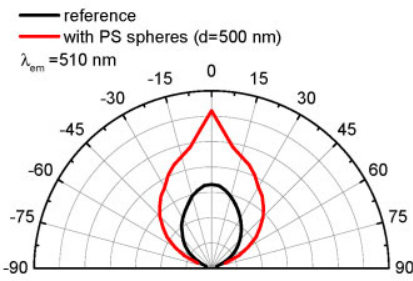

(c)

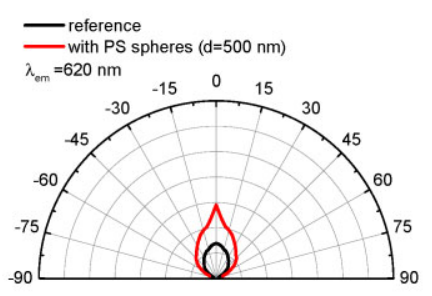

(d)

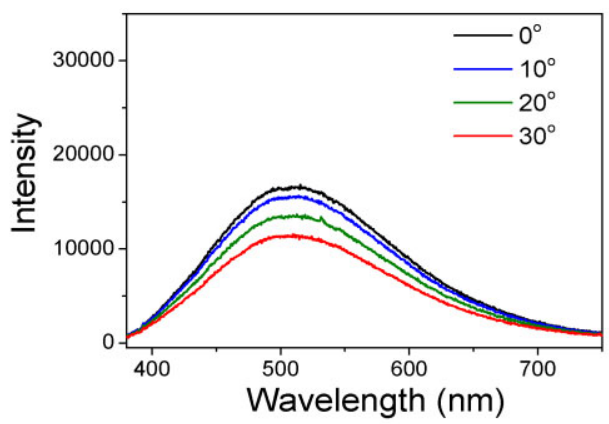

(b)

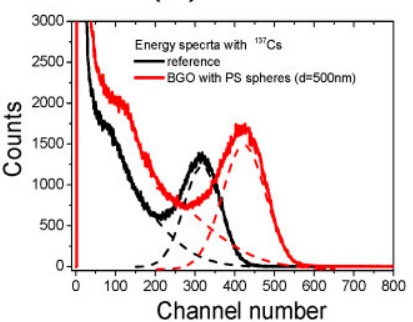

(e)

Figure 4 Emission spectra of BGO covered with a monolayer of PS nanospheres (a) and plain BGO (b) with different emergence angles. Angular profiles of emission at $510 \mathrm{~nm}$ (c) and $620 \mathrm{~nm}$ (d) for plain BGO (black lines) and BGO with nanospheres (red lines). The light output (e) under the excitation of $\gamma$-ray for plain BGO (black lines) and BGO with nanospheres (red lines). Gaussian fittings shown with dashed lines are used to analyze the energy resolution.

resolution [1]. The values of the energy resolution in the present study are obviously larger than the generally reported result of $9 \%$ for a BGO scintillator [27] because the samples in the present measurement were not surrounded with reflection layers and not coupled with optical glue, leading to a low collection efficiency.

The enhancement ratios of light extraction by PS nanospheres with different emerging angles are presented

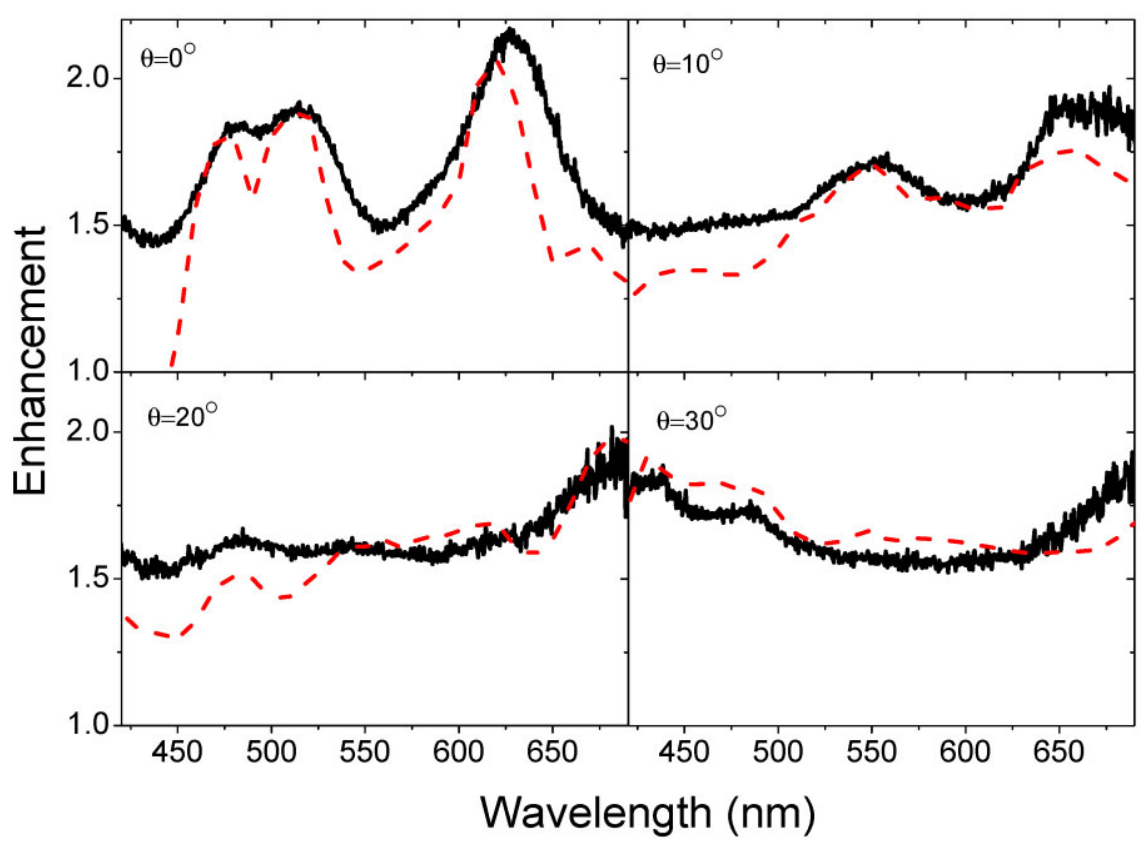

Figure 5 Enhancement ratios of light extraction by a monolayer of PS nanospheres for the emerging angles of $0^{\circ}, 10^{\circ}, 20^{\circ}$, and $30^{\circ}$. Black lines represent the experimental values and the red dashed lines represent the simulation values. 
in Fig. 5. The simulation results are in good agreement with the experimental values. In the normal direction (emerging angle of $0^{\circ}$ ), three wide bands peaked at 480, 513, and $627 \mathrm{~nm}$ appear. These bands exhibit wide bandwidths due to the characters of high energy leakage and low wavelength selectivity for low-order resonances of wavelength-scale resonators [15]. With the increase of emerging angle, the peak wavelengths of enhancement are changed, which is determined by photonic modes and orders of the monolayer of PS spheres. Although the peak wavelengths of the enhancement bands change with the increase of emergence angle, the wide band character still persists.

In the application of light extraction with broadband characteristics by an array of nanospheres, an important requirement is that only the low-order WGMs can be excited. WGMs with low order have low Q factors, and thus have a broadband characteristic that can match with the broad emission of BGO scintillator. Therefore, the optimal diameter $(500 \mathrm{~nm})$ should be close to the emission wavelength of BGO.

4 Summary We experimentally demonstrate broadband light-extraction enhancement of a BGO scintillator by an array of monolayer of PS nanospheres. The enhancement of wavelength- and angle-integrated luminescence by 72 and $68 \%$ compared with the reference sample can be achieved under the excitation of ultraviolet and $\gamma$-ray radiation, respectively. Furthermore, the enhancement is strongly dependent on the wavelength and the emergence angle. Combined with the simulation of the spatial distribution of electric-field intensity, it is revealed that the WGMs for the individual sphere and the periodic arrangement are responsible for the enhancement of light extraction, and WGMs play an important role in providing extra enhancement compared with a traditional two-dimensional photonic crystal. Low-order modes of WGMs with the character of high energy leakage and low wavelength selectivity are beneficial to the wideband emission of scintillators. The nature of the diffraction of the near field of WGMs by periodic structures allows control of the directionality of scintillation emission tobe obtained, which is critical for the application of radiation detection.

Acknowledgements This work was supported by the National Basic Research Program of China (Grant No. 2011CB922001), and the National Natural Science Foundation of China (Grant Nos. 11374229, 11179019, 11234010).

\section{References}

[1] A. Knapitsch, E. Auffray, C. W. Fabjan, J.-L. Leclercq, X. Letartre, R. Mazurczyk, and P. Lecoq, IEEE Trans. Nucl. Sci. 60, 2322 (2013).
[2] D. J. Brenner and E. J. Hall, New Engl. J. Med. 357, 2277 (2007).

[3] Y. Ohashi, N. Yasui, Y. Yokota, A. Yoshikawa, and T. Den, Appl. Phys. Lett. 102, 051907 (2013).

[4] N. Yasui, Y. Ohashi, T. Kobayashi, and T. Den, Adv. Mater. 24, 5464 (2012).

[5] A. Knapitsch, E. Auffray, C. W. Fabjan, J.-L. Leclercq, X. Letartre, R. Mazurczyk, and P. Lecoq, IEEE Trans. Nucl. Sci. 59, 2334 (2012).

[6] P. Pignalosa, B. Liu, H. Chen, H. Smith, and Y. Yi, Opt. Lett. 37, 2808 (2012).

[7] W. J. Hyun, H. K. Lee, S. S. Oh, O. Hess, C.-G. Choi, S. H. Im, and O. O. Park, Adv. Mater. 23, 1846 (2011).

[8] J. Jonathan, J. Wierer, A. David, and M. M. Megens, Nature Photon. 3, 163 (2009).

[9] Y. R. Do, Y. C. Kim, Y.-W. Song, C.-O. Cho, H. Jeon, Y.-J. Lee, S.-H. Kim, and Y.-H. Lee, Adv. Mater. 15, 1214 (2003).

[10] S. Fan, P. R. Villeneuve, J. D. Joannopoulos, and E. F. Schubert, Phys. Rev. Lett. 78, 3294 (1997).

[11] J. K. Kim, S. Chhajed, M. F. Schubert, E. F. Schubert, A. J. Fischer, M. H. Crawford, J. Cho, H. Kim, and C. Sone, Adv. Mater. 20, 801 (2008).

[12] C.-Y. Fang, Y.-L. Liu, Y.-C. Lee, H.-L. Chen, D.-H. Wan, and C.-C. Yu, Adv. Mater. 23, 1412 (2013).

[13] J. H. Son, J. U. Kim, Y. H. Song, B. J. Kim, C. J. Ryu, and J.-L. Lee, Adv. Mater. 24, 2259 (2012).

[14] H. T. Miyazaki, H. Miyazaki, K. Ohtaka, and T. Sato, J. Appl. Phys. 87, 7152 (2000).

[15] Y. Yao, J. Yao, V. K. Nnarasimhan, Z. Ruan, C. Xie, S. Fan, and Y. Cui, Nature Commun. 3, 664 (2012).

[16] Z. Zhu, B. Liu, C. Cheng, Y. Yi, W. Guo, S. Huang, H. Chen, M. Gu, C. Ni, and X. Liu, Opt. Mater. 35, 2343 (2013).

[17] Z. Zhu, B. Liu, C. Cheng, Y. Yi, H. Chen, and M. Gu, Appl. Phys. Lett. 102, 071909 (2013).

[18] G. Kang, H. Park, D. Shin, S. Baek, M. Choi, D.-H. Yu, K. Kim, and W. J. Padilla, Adv. Mater. 25, 2617 (2013).

[19] J. Grandidier, R. A. Weitekamp, M. G. Deceglie, D. M. Callahan, C. Battaglia, C. R. Bukowsky, C. Ballif, R. H. Grubbs, and H. A. Atwater, Phys. Status Solidi A 210, 255 (2013).

[20] J. Grandidier, D. M. Callahan, J. N. Munday, and H. A. Atwater, Adv. Mater. 23, 1272 (2011).

[21] J. F. Galisteo-López, M. Ibisate, R. Sapienza, L. S. FroufePérez, Á. Blanco, and C. López, Adv. Mater. 23, 30 (2011).

[22] X. Ye and L. Qi, Nano Today 6, 608 (2011).

[23] M. López-García, J. F. Galisteo-López, C. López, and A. GarcíaMartín, Phys. Rev. B 85, 235145 (2012).

[24] B. M. Möller, U. Woggon, and M. V. Artemyev, Opt. Lett. 30, 2116 (2005).

[25] Y. Hara, T. Mukaiyama, K. Takeda, and M. Kuwata-Gonokami, Phys. Rev. Lett. 94, 203905 (2005).

[26] E. F. Schubert, Light-Emitting Diodes, second ed. (Cambridge University Press, New York, 2006), p. 94.

[27] W. Chewpraditkul, K. Sreebunpeng, M. Nikl, J. A. Mares, K. Nejezchleb, A. Phunpueok, and C. Wanarak, Radiat. Meas. 47, 1 (2012). 